\title{
Impact of dietary manganese on experimental colitis in mice
}

\author{
Eun-Kyung Choi $^{1}$ | Luisa Aring $^{1}$ | Nupur K. Das ${ }^{2}$ | Sumeet Solanki ${ }^{2}$ | \\ Naohiro Inohara $^{3}$ | Shigeki Iwase ${ }^{4}$ | Linda C.Samuelson ${ }^{2}$ | Yatrik M. Shah ${ }^{2}$ | \\ Young Ah Seo ${ }^{1}$
}

\author{
${ }^{1}$ Department of Nutritional Sciences, \\ University of Michigan School of Public \\ Health, Ann Arbor, MI, USA \\ ${ }^{2}$ Department of Molecular and Integrative \\ Physiology, University of Michigan Medical \\ School, Ann Arbor, MI, USA \\ ${ }^{3}$ Department of Pathology, University of \\ Michigan Medical School, Ann Arbor, MI, \\ USA \\ ${ }^{4}$ Department of Human Genetics, \\ University of Michigan, Ann Arbor, MI, \\ USA

\section{Correspondence} \\ Young Ah Seo, Department of Nutritional \\ Sciences, University of Michigan School of \\ Public Health, Ann Arbor, MI 48109 USA. \\ Email: youngseo@umich.edu

\section{Funding information} \\ HHS I National Institutes of Health (NIH), \\ Grant/Award Number: R00 ES024340, \\ R01 DK095201, R01 DK118023 and R01 \\ NS089896; Crohn's and Colitis Foundation \\ (Crohn's \& Colitis Foundation); HHS I \\ NIH I National Institute of Diabetes and \\ Digestive and Kidney Diseases (NIDDK), \\ Grant/Award Number: 5P30DK034933
}

\begin{abstract}
Diet plays a significant role in the pathogenesis of inflammatory bowel disease (IBD). A recent epidemiological study has shown an inverse relationship between nutritional manganese (Mn) status and IBD patients. Mn is an essential micronutrient required for normal cell function and physiological processes. To date, the roles of $\mathrm{Mn}$ in intestinal homeostasis remain unknown and the contribution of Mn to IBD has yet to be explored. Here, we provide evidence that $\mathrm{Mn}$ is critical for the maintenance of the intestinal barrier and that Mn deficiency exacerbates dextran sulfate sodium (DSS)-induced colitis in mice. Specifically, when treated with DSS, Mn-deficient mice showed increased morbidity, weight loss, and colon injury, with a concomitant increase in inflammatory cytokine levels and oxidative and DNA damage. Even without DSS treatment, dietary Mn deficiency alone increased intestinal permeability by impairing intestinal tight junctions. In contrast, mice fed a Mn-supplemented diet showed slightly increased tolerance to DSS-induced experimental colitis, as judged by the colon length. Despite the well-appreciated roles of intestinal microbiota in driving inflammation in IBD, the gut microbiome composition was not altered by changes in dietary $\mathrm{Mn}$. We conclude that Mn is necessary for proper maintenance of the intestinal barrier and provides protection against DSS-induced colon injury.
\end{abstract}

\section{K E Y W O R D S}

colitis, gut microbiota, inflammatory bowel disease, manganese, tight junction

\section{1 | INTRODUCTION}

Inflammatory bowel disease (IBD) manifests as two major types-Crohn's disease and ulcerative colitis-which are relapsing chronic inflammatory disorders of the intestine. The incidence of IBD is rapidly increasing worldwide, with over 3 million individuals in the United States with 70000 new cases diagnosed each year. ${ }^{1}$ A number of common gastrointestinal disorders, including IBD, share a common impairment of the intestinal barrier function. ${ }^{2}$ This barrier consists of epithelial cells with intercellular junctions that link adjacent cells and seal the intercellular spaces. ${ }^{2}$ The barrier protects against the penetration of harmful solutes, microorganisms, toxins, and luminal antigens into the body, while acting as a selective

\footnotetext{
Abbreviations: 8-OHdG, 8-hydroxy-2'-deoxyguanosine; BC, Bray-Curtis dissimilarity index; DSS, dextran sulfate sodium; ELISA, enzyme-linked immunosorbent assay; FITC, fluorescein isothiocyanate; GWAS, genome-wide association studies; IBD, inflammatory bowel disease; IL, interleukin; LDA, Linear discriminant analysis; LEfSe, linear discriminant analysis effect size; Mn, manganese; MnSOD, manganese superoxide dismutase; NMDS, Non-metric multidimensional scaling; OTU, operational taxonomic unit; PBS, phosphate-buffered saline; PCR, polymerase chain reaction; ROS, reactive oxygen species; TNF, tumor necrosis factor; ZO-1, zonula occludens-1.
} 
filter that permits the absorption of essential nutrients, electrolytes, and water from the intestinal lumen and their release into the circulation. ${ }^{2}$ Impairment of the barrier function permits the invasion of luminal bacteria into the lamina propria, thereby triggering an immune response and intestinal inflammation. ${ }^{2}$ At present, no cure exists for IBD and treatment options are limited.

Complex interactions between genetic, environmental, microbial, and immune factors are believed to contribute to IBD disorders. ${ }^{3}$ The recent identification of $>200$ susceptibility loci that confer vulnerability to IBD in genome-wide association studies ${ }^{4}$ has underscored the significant role of genetics in IBD. However, while genetic risk factors clearly play a role, genetics contribute to only a small fraction of the disease risk. Environmental factors are also suspected as triggers and these may explain the weakly penetrant disease development. Indeed, dietary nutrients are now implicated in numerous epidemiological and experimental studies as prominent factors that may play critical roles in IBD. ${ }^{5}$ Macronutrients, such as glucose and lipids, play a pivotal role in the development of $\mathrm{IBD}^{5}$; however, evidence now also points to a role for micronutrients in both disease development and treatment. To date, many micronutrients (eg, vitamins A, C, D, and E) and trace minerals (eg, iron ( $\mathrm{Fe})$, zinc $(\mathrm{Zn})$, and selenium $(\mathrm{Se})$ ) have been implicated in the pathogenesis of IBD. ${ }^{6}$ However, one essential micronutrient, manganese (Mn), has yet to be explored as a risk factor for IBD.

Mn deficiency is rare in humans due to its availability in numerous dietary sources. Mn is abundant in plant-based foods, such as whole grains, legumes, rice, nuts, and vegetables, although it is relatively deficient in animal sources, including meat, fish, poultry, eggs, and dairy products. ${ }^{7}$ However, low Mn levels have been reported in IBD patients. For example, a recent epidemiological survey measured hair micronutrient levels in pediatric patients newly diagnosed with Crohn's disease and ulcerative colitis from 2012 to $2016 .{ }^{8}$ This study identified that Fe $(P=.033)$, Se $(P=.017)$, and $\mathrm{Mn}(P=.009)$ were significantly lower in patients with Crohn's disease and ulcerative colitis than in healthy controls, implicating insufficiency in these trace minerals, including $\mathrm{Mn}$, as potential risk factors for IBD.

$\mathrm{Mn}$ is an essential micronutrient required for normal cell function and for a variety of physiological processes, such as bone growth, regulation of blood sugar and cellular energy levels, blood coagulation and hemostasis, and defense against reactive oxygen species (ROS). ${ }^{9} \mathrm{Mn}$ directs cellular processes by acting as a cofactor in the reactions catalyzed by numerous enzymes, including Mn superoxide dismutase (MnSOD), arginase, xanthine oxidase, galactosyltransferase, pyruvate decarboxylase, and glutamine synthetase. ${ }^{9}$ Some of the Mnrelated cellular processes, such as defense against ROS, have been implicated in IBD, suggesting that Mn may play a role in IBD pathogenesis. The goal of the present study was to determine the contribution of dietary Mn to IBD in a mouse model of dextran sulfate sodium (DSS)-induced colitis.

\section{MATERIALS AND METHODS}

\subsection{Animals and treatment conditions}

Wild-type (WT) C57BL/6 mice aged 3-4 weeks were purchased from Jackson Laboratory and maintained on a metalbasal diet containing $35 \mathrm{mg} \mathrm{Mn} / \mathrm{kg}$ (TD120518, Harlan Teklad, Indianapolis, IN, USA) (Table S1), as previously described. ${ }^{10,11}$ The trace element levels in the diet were as recommended by the American Institute of Nutrition. ${ }^{12}$ For dietary $\mathrm{Mn}$ alterations, the mice were fed either Mndeficient, Mn-adequate, or Mn-supplemented diets $(<0.01$, 35, and $300 \mathrm{ppm} \mathrm{Mn,} \mathrm{respectively;} \mathrm{Harlan} \mathrm{Teklad).} \mathrm{The}$ actual Mn concentrations of the diets were 0-0.5 ppm Mn (Mn-deficient), 35-35.5 ppm Mn (Mn-adequate), and 300$301 \mathrm{ppm}$ Mn (Mn-supplemented), as determined previously by inductively coupled plasma mass spectrometry (ICP-MS). ${ }^{13,14}$ For colitis induction, mice were provided with water containing 3\% (w/v) DSS for 6 days (inflammatory phase). The mice were then placed on regular drinking water for 7 days (recovery phase). All mice in the same experiment were from an age-matched, co-housed cohort. This study was performed in strict accordance with the recommendations in the Guide for the Care and Use of Laboratory Animals of the National Institutes of Health (Bethesda, MD, USA). The protocol (protocol number: PRO00008963) was approved by the University Committee on Use and Care of Animals (UCUCA) at the University of Michigan.

\section{2 | RNA isolation and RT-PCR}

Total RNA was isolated from colon mucosal cells using TRIzol reagent (Invitrogen; Thermo Fisher Scientific, Inc., Waltham, MA, USA) following the manufacturer's instructions. Purified RNA was then reverse-transcribed with SuperScript III FirstStrand Synthesis System (Invitrogen; Thermo Fisher Scientific, Inc.). The RT-PCR was performed using the Power SYBRGreen PCR Master Mix (Applied Biosystems; Thermo Fisher Scientific, Inc.). GAPDH was used for normalization of the mRNA. The primers used for qPCR are listed in Table S2 and were all purchased from Integrated Genomics Technologies.

\subsection{Immunoblot analysis}

Colonic mucosal cells were scraped and lysed in RIPA buffer containing protease inhibitors (Roche, Basel, Switzerland; Cat. No. 11836153001). Protein concentrations were 
determined with the Bradford assay. Samples (30-50 $\mu \mathrm{g})$ were separated by electrophoresis and transferred to a nitrocellulose membrane (Bio-Rad, Hercules, CA, USA; Cat. No. 1620115). The membrane was immunoblotted with anti-ZO-1 antibody (Santa Cruz, Dallas, Texas, USA; Cat. No. 33725), anti-ZO-2 antibody (Santa Cruz; Cat. No. 515115), anti-Occludin antibody (Santa Cruz; Cat. No. 133256), anti-Claudin 2 antibody (Santa Cruz; Cat. No. 293233), and anti-mouse actin (Proteintech, Rosemont, IL USA; Cat. No. 60008-1-Ig). The blots were visualized with infrared anti-mouse or anti-rat secondary antibodies, using an LI-COR Odyssey fluorescent western blotting system (LI-COR Biosciences, Lincoln, NE, USA). Protein expression was quantified by densitometry (Image Studio Lite; LI-COR).

\section{4 | Histology and immunohistochemical staining}

Colon tissues were fixed in $4 \%$ paraformaldehyde at $4{ }^{\circ} \mathrm{C}$ overnight. $\mathrm{H} \& \mathrm{E}$ analysis was performed in paraffin-embedded tissue sections (5 microns). The histological scoring was performed by a pathologist who blindly assessed the degree of surface epithelial loss, crypt destruction, and inflammatory cell infiltration into the mucosa, resulting in a score from 0 to 12 .

\section{5 | Trace element analysis}

Tissue samples were analyzed for metals by ICP-MS. Briefly, tissue samples taken from the mice were digested with $2 \mathrm{~mL} / \mathrm{g}$ total wet weight nitric acid (BDH ARISTAR ULTRA) for 24 hours and then digested with $1 \mathrm{~mL} / \mathrm{g}$ total wet weight hydrogen peroxide (BDH Aristar ULTRA) for 24 hours at room temperature. Specimens were preserved at $4^{\circ} \mathrm{C}$ until quantification of metals. Ultrapure water was used for the final sample dilution.

\subsection{Manganese superoxide dismutase assay}

Mitochondrial MnSOD activity was determined using a Superoxide Dismutase Assay Kit (Cayman Chemical, Ann Arbor, MI, USA) as described previously. ${ }^{11,13}$ Briefly, the colonic mucosal cells were scraped into ice-cold MB buffer (10 mM HEPES, pH 7.5, $210 \mathrm{mM}$ mannitol, $70 \mathrm{mM}$ sucrose, and $1 \mathrm{mM}$ EDTA) and then homogenized with a Dounce glass homogenizer. The cell extract was centrifuged at $1000 \mathrm{~g}$ for 5 minutes at $4^{\circ} \mathrm{C}$ and the mitochondrial fraction was pelleted by centrifugation at $12000 \mathrm{~g}$ for 12 minutes at $4^{\circ} \mathrm{C}$. MnSOD activity was determined in the presence of $2 \mathrm{mM}$ potassium cyanide to inhibit $\mathrm{Cu} / \mathrm{Zn}-\mathrm{SOD}$.

\section{$2.7 \quad$ Isoprostane analysis}

Isoprostane was chosen as a marker of oxidative damage and measured by a competitive enzyme-linked immunosorbent assay (ELISA) for one of the isoprostanes 8-iso Prostaglandin F2 $\alpha$ (8-iso-PGF) with a commercial kit (Cayman Chemical, Ann Arbor, MI). The assay was based on the competition between 8-iso-PGF and 8-isoprostane-acetylcholinesterase (AChE) conjugate for a limited number of binding sites in each ELISA plate well. The concentration of 8-iso-PGF is inversely proportional to the number of binding sites available, whereas $\mathrm{AChE}$ is held constant. The assays were performed as previously described. ${ }^{13}$

\section{8 | Measurement of oxidative DNA damage}

Serum oxidative DNA damage was measured using a commercial 8-hydroxy-2-deoxyguanosine (8-OHdG) ELISA assay kit (Cayman Chemical, Ann Arbor, MI) according to the manufacturer's protocol. Serum samples were diluted $1: 50$ prior to analysis.

\section{9 | Hydrogen peroxide assay}

Tissue hydrogen peroxide levels were measured using a hydrogen peroxide assay kit (Abcam, Cambridge, United Kingdom), according to the instructions supplied by the manufacturer.

\subsection{0 | Microbiota analysis}

Fecal DNA was extracted using an EZNA stool DNA kit (Omega Bio-tek). Microbiota composition was determined by sequencing the V4 region of the $16 \mathrm{~S}$ rRNA gene $(\approx 250 \mathrm{bp})$ from the $5^{\prime}$ and $3^{\prime}$ ends with Illumina MiSeq and the resulting reads were assembled and analyzed by Mothur, as described previously. ${ }^{15}$ The OTUs were classified into taxonomic phylotypes at $>97 \%$ identity. The Shannon, Chao, and Simpson diversity indexes, Shannon evenness index, $\theta_{\mathrm{YC}}$, and BrayCurtis dissimilarity indexes were calculated by Mothur, whereas the operational taxonomic unit (OTU) richness was calculated by Microsoft Excel. An NMDS plot of $\beta$-diversity with the minimal stress value was generated and the LEfSe Linear discriminant analysis (LDA) values of the OTUs and the false discovery rate between two groups were determined by Mothur. Comparative marker selection was used to complement the LEfSe analysis via GENE-E (https://www.broad institute.org/cancer/software/GENE-E/) using the default settings and 10,000 permutations. 


\subsection{Intestinal permeability assay}

Fluorescein isothiocyanate (FITC)-dextran (4 kDa, FD4; Sigma, St. Louis, MO, USA) was used as an indicator to examine barrier function. Briefly, mice were gavaged with FITC-dextran (40 mg/100 g body weight). Four hours later, the fluorescence intensity of the serum samples was measured. Serum was collected and the fluorescence intensity of each sample (excitation, $485 \mathrm{~nm}$; emission, $525 \mathrm{~nm}$ ) was measured using a BioTek Synergy microplate reader (BioTek Instruments, Winooski, VT). The FITC-dextran concentrations were determined from standard curves generated by the serial dilution of FITC-dextran.

\subsection{2 | Statistical analysis}

Statistical analyses were performed using GraphPad Prism software version 8 (GraphPad Software Inc., La Jolla, CA, USA). PAST 3.22 was used for PERMANOVA calculation based on 9999 permutations. The Shapiro-Wilk normality test was used to test the assumption of normal distribution. Differences between two groups were evaluated using a two-tailed unpaired t test or the Mann-Whitney $U$ test for normally or non-normally distributed datasets, respectively. The comparison of more than two groups was performed with one-way ANOVA, followed by the Dunnett's multiple comparisons test for normally distributed datasets or by either the Kruskal-Wallis or Friedman test, both followed by the Dunn's multiple comparisons test, for data with nonnormal distributions. For highly repetitive comparisons between two groups, we also performed false positive rate analysis using the metastats command in Mothur, after the removal of the OTUs that were not included in the tested dataset. PERMANOVA was used for the analysis of the Bray-Curtis indexes for Figure 5. Differences at $P<.05$ and $\mathrm{q}<0.05$ were considered statistically significant.

\section{3 | RESULTS}

\section{1 | Mn deficiency exacerbates intestinal injury after DSS treatment}

We explored the roles of Mn in colitis by first investigating whether dietary Mn alters the susceptibility to DSS-induced injury, which is characterized by robust epithelial denudation and inflammation. ${ }^{16}$ DSS damages the epithelial monolayer that lines the large intestine, thereby allowing the proinflammatory intestinal contents to pass through the intestinal barrier. ${ }^{16}$ Mice were fed with either a Mn-deficient ( $<0.01 \mathrm{mg} \mathrm{Mn} \mathrm{per} \mathrm{kilogram} \mathrm{of} \mathrm{diet)} \mathrm{or} \mathrm{Mn-adequate} \mathrm{(35} \mathrm{mg}$ $\mathrm{Mn} / \mathrm{kg}$ ) diet for 14 days, followed by treatment with DSS
(Figure 1A). Two weeks on the Mn-deficient diet was sufficient to induce intestinal Mn deficiency, as assessed by ICP-MS of the duodenum and colon tissues. The mice fed with Mn-deficient diet showed an $86-96 \%$ reduction in Mn levels in the duodenum and colon (Figure 1B,C). The levels of other trace elements, including $\mathrm{Fe}, \mathrm{Zn}$, and $\mathrm{Cu}$, were not altered in these tissues (Figure 1B,C). Heavy metals, such as cadmium and lead, were not detected in these samples. We found no differences in body weights or food intake during the 2-week experimental period in the mice fed Mn-deficient and Mn-adequate diets (Figure 1D,E). These findings demonstrate that 2 weeks of dietary Mn deficiency influenced Mn levels in the intestines without affecting other trace element levels.

Having established a condition that achieved dietary Mn deficiency, we treated mice fed either Mn-deficient or Mn-adequate diets with 3\% DSS in the drinking water to induce colitis (inflammatory phase) (Figure 1A). The mice were then placed on regular drinking water for 7 days (recovery phase) (Figure 1A). During the monitoring phases, the mice continued to receive the same Mn diets. The mice fed with Mn-adequate diet and subjected to DSS treatment underwent profound and sustained weight loss, indicative of increased susceptibility to colitis (Figure 2A). Notably, the Mn-deficient mice displayed an even more enhanced bodyweight loss when compared to the Mn-adequate mice (Figure 2A). When placed back on regular water, the Mn-adequate mice recovered their original body weights, whereas the Mn-deficient mice did not show any recovery and continued to lose body weight until the experimental endpoint (Figure 2A). Most of the Mn-adequate mice survived the DSS treatment, whereas approximately $60 \%$ of the Mn-deficient mice died by Day 13 (Figure 2B). The Mn-deficient mice also showed an approximately 13\% decrease in colon length when compared with the Mn-adequate mice (Figure 2C,D). The ratio of the spleen to body weight was higher in the Mn-deficient mice than in the Mn-adequate mice, although this trend did not reach statistical significance $(P=.055$, Figure 2E). These data demonstrate that dietary Mn deficiency exacerbates intestinal injury in this mouse model of DSS-induced colitis.

\section{2 | Elevated inflammation in Mn-deficient mice in response to DSS}

The administration of DSS in the drinking water causes intestinal epithelial injury and robust inflammatory response. ${ }^{16}$ Histological analysis of the colons of the Mndeficient mice revealed increased tissue damage, including severe surface epithelial loss, crypt destruction, massive infiltration of inflammatory cells, and extensive degeneration of the epithelial structure (Figure $3 \mathrm{~A}$ ). The total 
(A)

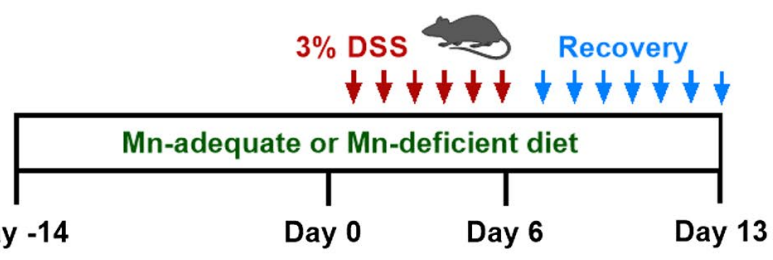

(B)

Duodenum
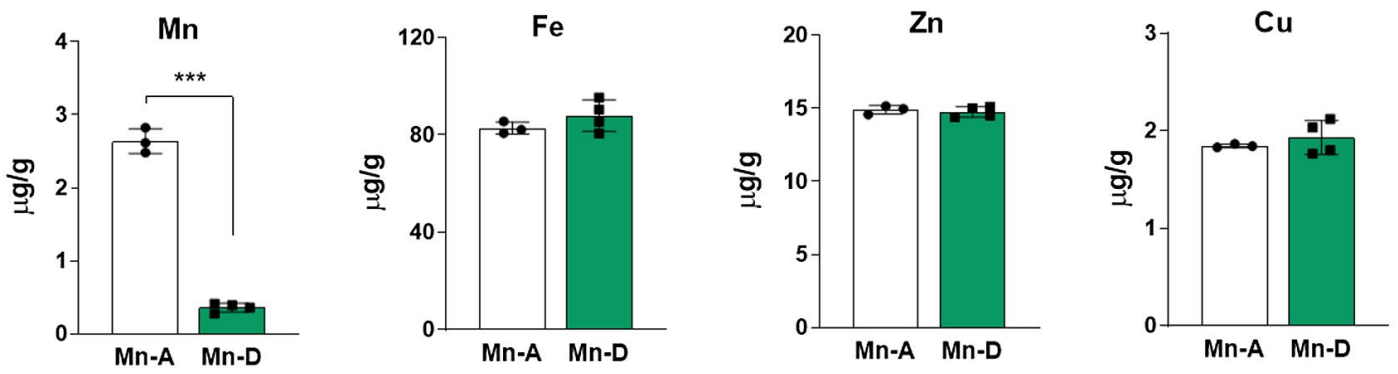

(C)

Colon
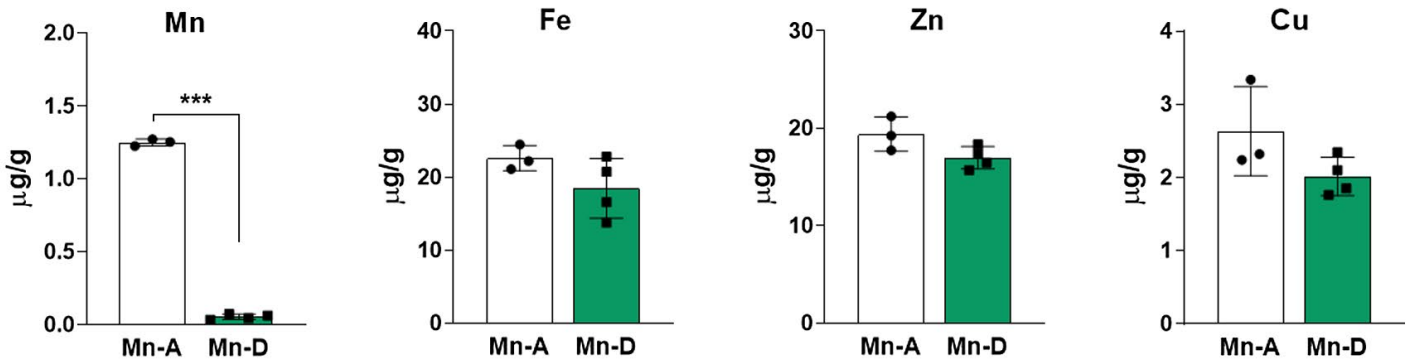

(D)

(E)
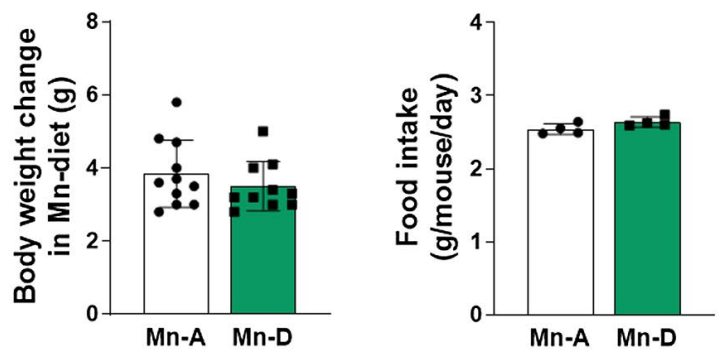

F I G URE 1 Dietary Mn deficiency reduces intestinal Mn levels. A, Schematic of the DSS-induced colitis model. Mice were fed with either Mn-deficient (Mn-D) or Mn-adequate (Mn-A) diets ( 0 and $35 \mathrm{mg} / \mathrm{kg} \mathrm{Mn}$, respectively). For colitis induction, mice were provided with drinking water containing 3\% (w/v) DSS for 6 days (inflammatory phase). The mice were then provided with regular drinking water for 7 days (recovery phase). B and C, Levels of the trace elements manganese $(\mathrm{Mn})$, iron (Fe), zinc $(\mathrm{Zn})$, and copper $(\mathrm{Cu})$ were measured by ICP-MS in the (B) duodenum and $(C)$ colon of mice ( $n=3-4$ per group). D, Body weight changes ( $n=10-11$ per group) and (E) daily food intake ( $n=4$ per group) were assessed. Data represent means \pm SEM. $* * * P<.001$

pathological score, which takes into account the surface epithelial loss, crypt destruction, and inflammatory cell infiltration into the mucosa, was significantly higher in the colons of Mn-deficient mice than in the colons of Mnadequate mice (Figure 3B). Chemokine and cytokine signaling were also aberrantly regulated in the Mn-deficient mice in response to intestinal injury. The Mn-deficient mice showed significantly higher expression of chemokines Ccl2 and Cxcll and of inflammatory cytokines Tnfo, Il6, $I 11 \beta$, and $I l 10$ (Figure 3C). Taken together, these data demonstrate that dietary Mn deficiency exacerbates intestinal inflammation in DSS-induced colitis in mice. 
(A)

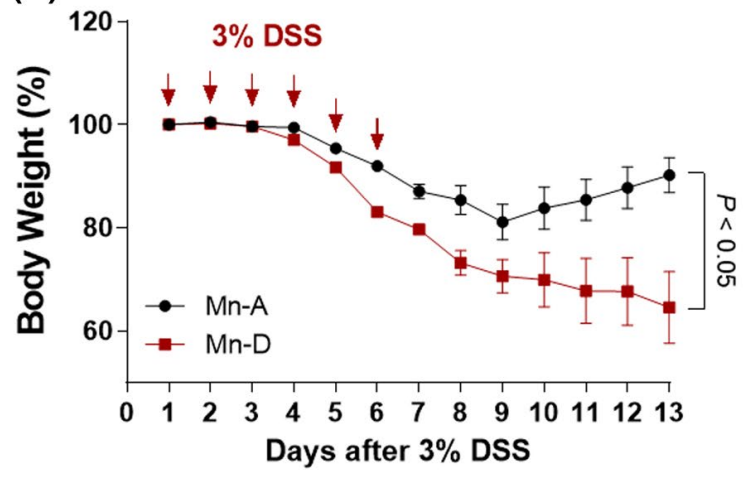

(B)

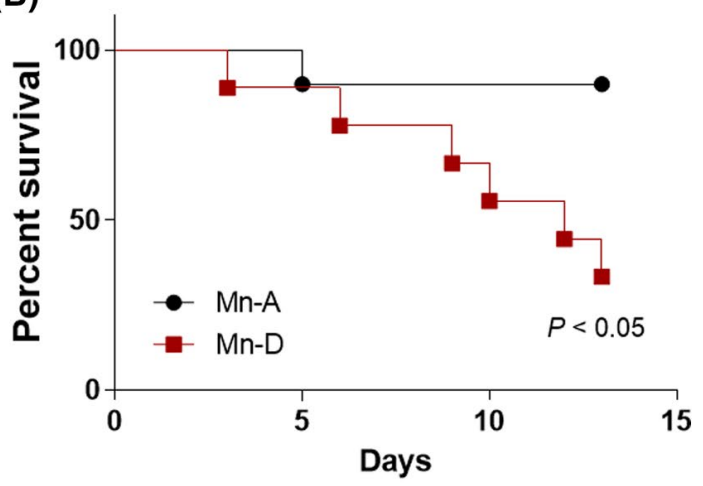

(C)

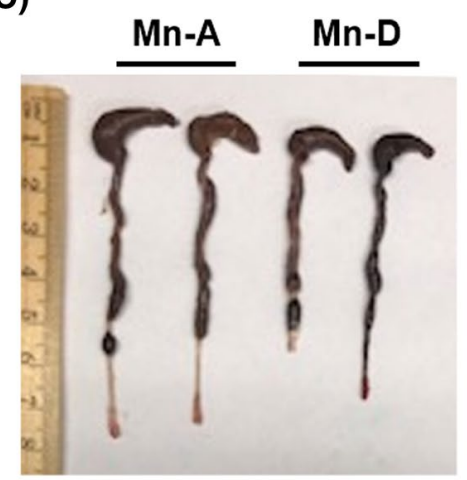

(D)

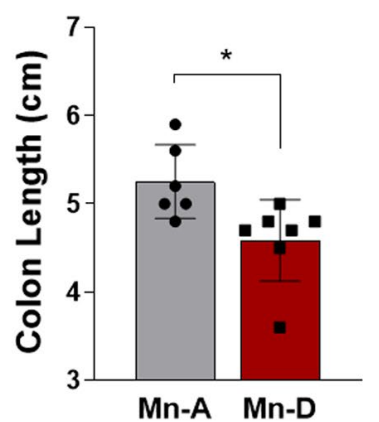

(E)

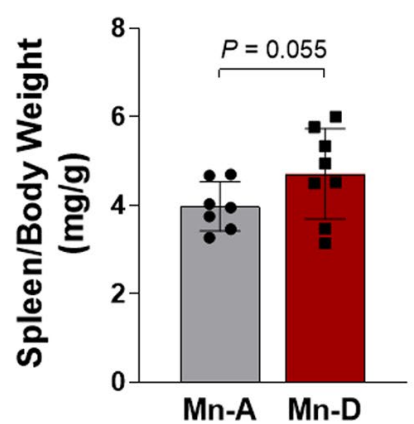

F I G U RE 2 Mn deficiency exacerbates intestinal injury in a mouse model of DSS-induced colitis. A, Body weight changes over time. Mice were provided with drinking water containing $3 \%$ DSS (w/v) for 6 days, followed by 7 days of regular drinking water. Body weight was measured over 13 days ( $\mathrm{n}=15$ per group). B, Survival percentage (\%) over time. C, Gross morphology of the large intestine. D, Colon length, and E, spleen/ body weight ratios at 6 days after the DSS treatment in mice fed Mn-adequate (Mn-A) or Mn-deficient (Mn-D) diets ( $\mathrm{n}=6-8$ per group). Data represent means \pm SEM. $* P<.05$

\section{3 | Intestinal permeability is increased in Mn-deficient mice in response to DSS}

Increased intestinal permeability caused by dysfunctions of the intestinal barrier is a key cellular mechanism underlying DSS-induced colitis and human IBD. ${ }^{16}$ We measured intestinal permeability in our mouse colitis model using FITC-dextran. Notably, the serum FITC-dextran levels on Day 6 of DSS treatment were significantly higher in the Mndeficient mice than in the Mn-adequate mice, indicating an increased intestinal permeability associated with Mn deficiency (Figure 4A).

One possible mechanism that might mediate this effect could be an impairment of the mucosal barrier. ${ }^{17}$ DSS treatment is known to decrease the expression of tight junction proteins, which then leads to subsequent increases in the permeability of the mucosal barrier. ${ }^{16}$ Therefore, we examined the expression of the key components of colon epithelial tight junctions, as these structures can directly affect intestinal permeability. RT-PCR quantification showed substantial reductions in the gene expression of tight junction components (Zo1, Zo2, Cldn2, Cldn3, and $O c l n$ ) in the colon mucosa of the Mn-deficient mice after DSS treatment (Figure 4B). In contrast, the expression of Cldn4, 5, 7, 12, and 15 did not change, suggesting a selective impact of Mn deficiency on tight-junction gene expression.

We also examined colon epithelial adherens junctions, as these indirectly regulate the integrity of tight junctions. ${ }^{18}$ Among the examined genes that encode adherens junction components, the expression of the cadherin gene $C d h l$ was reduced in Mn-deficient mice after DSS treatment, whereas the level of Nectinl was unchanged (Figure 4C). Further analysis by immunoblotting revealed diminished levels of Zonula occludens1 (ZO1), ZO2, Claudin2 (CLDN2), and Occludin $(\mathrm{OCLN})$ in Mn-deficient animals (Figure 4D,E). These data demonstrated that Mn deficiency reduced the expression of a subset of tight junction and adherens junction components in the colon and indicated that these changes may underlie the increased intestinal permeability observed in DSS-treated mice. These findings suggest that Mn deficiency exacerbates the impairment of the intestinal barrier in the DSS-induced colitis model. 
(A)
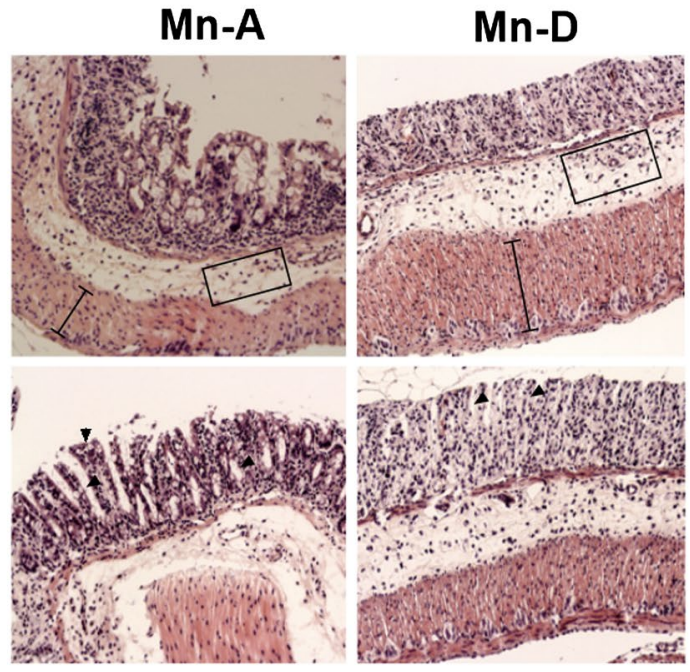

has 20
(B)

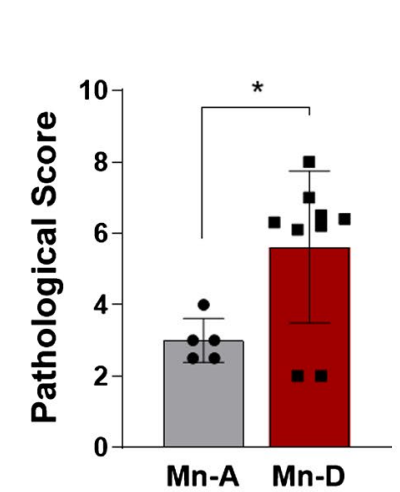

(C)

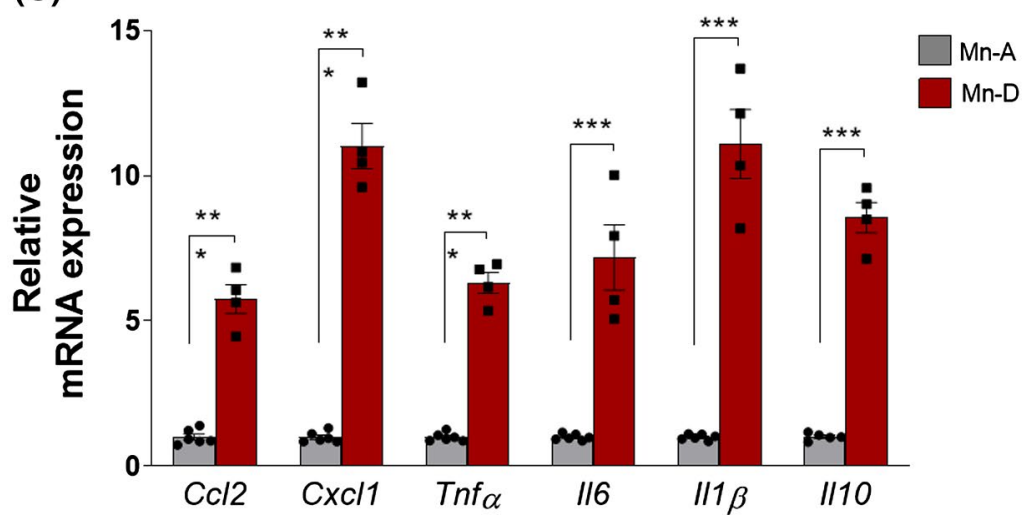

F I G U R E 3 Intestinal injury and inflammation are enhanced in Mn-deficient mice after DSS injury. A, Representative hematoxylin/eosin (H\&E) staining of colons and (B) histological scores in Mn-adequate (Mn-A) or Mn-deficient (Mn-D) mice at 13 days after the DSS treatment ( $n=5-9$ per group). C, RT-PCR quantification of proinflammatory cytokines and chemokines in colon mucosa on Day 6 after DSS treatment ( $\mathrm{n}=4-6$ per group). Data represent means \pm SEM. $* P<.05 ; * * * P<.001$

\section{4 | Mn deficiency induces oxidative damage after DSS treatment}

DSS-induced intestinal injury results in an increase in oxidative stress, which is at least partly attributable to immune cell infiltration and the release of inflammatory mediators. ${ }^{19}$ Increases in immune cell infiltration and inflammation were evident in the Mn-deficient mice after DSS treatment (Figure 3). Therefore, we next determined whether a Mn-deficient diet might reduce the activity of a specific Mn-requiring enzyme, manganese superoxide dismutase (MnSOD). MnSOD is a potent antioxidant enzyme located in the mitochondria. We first isolated mitochondria from the colons of DSS-treated mice fed either Mn-deficient or Mn-adequate diets and measured MnSOD activity. The MnSOD activity after DSS treatment was approximately $57 \%$ lower in the colons of Mn-deficient mice than in Mn-adequate mice (Figure 4F).
We then determined whether the observed reduction in cellular MnSOD activity involved altered production of ROS. Measurement of $\mathrm{H}_{2} \mathrm{O}_{2}$ levels in colon extracts revealed significantly higher $\mathrm{H}_{2} \mathrm{O}_{2}$ levels in the colons of $\mathrm{Mn}$-deficient mice than in Mn-adequate mice (Figure 4G). We also measured oxidative stress by determining the levels of two biomarkers: 8-isoprostane and 8-hydroxy-2'-deoxyguanosine (8-OHdG). The 8-isoprostane biomarker is a prostaglandin (PG)-F2-like compound produced by the free radical-catalyzed peroxidation of arachidonic acid. ${ }^{20}$ The levels of 8-isoprostane after DSS administration were significantly higher in the Mn-deficient than in the Mnadequate mice (Figure $4 \mathrm{H}$ ), indicating that $\mathrm{Mn}$ protects against lipid peroxidation in this mouse colitis model. The 8-OHdG biomarker is excreted during the repair of DNA damage, thereby providing a quantitative index of oxidative DNA damage. ${ }^{21}$ The 8 -OHdG levels were significantly increased in the serum of Mn-deficient mice after DSS injury 
(A)

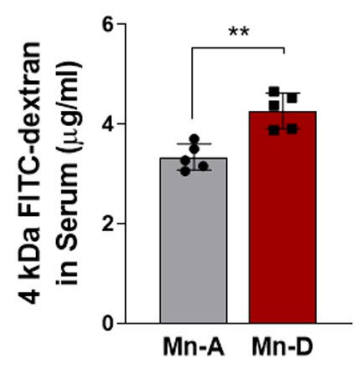

(C)

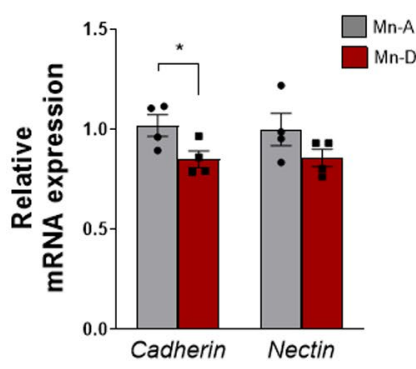

(F)

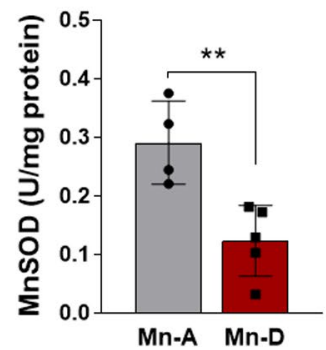

(B)

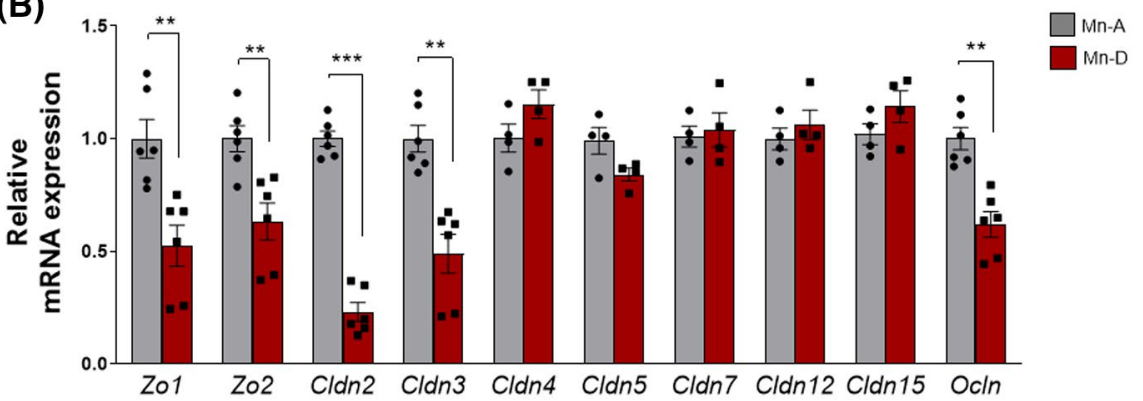

(D)

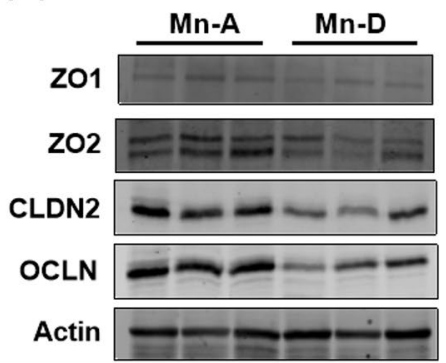

(E)

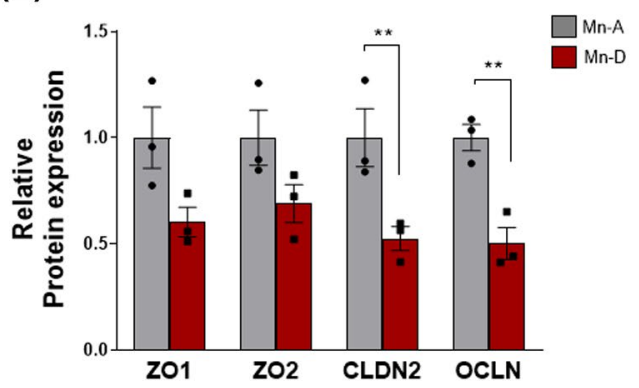

(G)

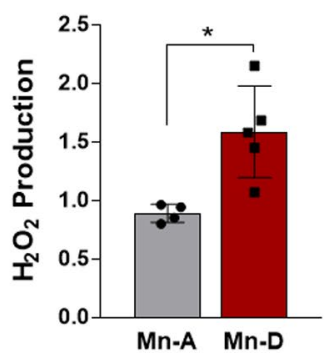

(H)

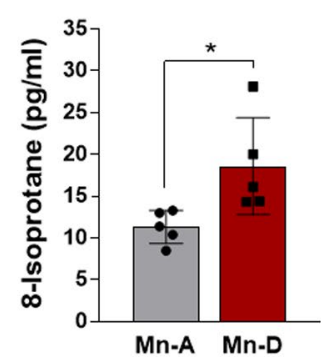

(I)

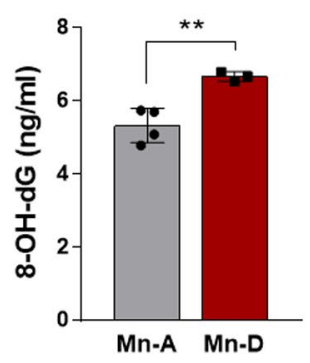

F I G U RE 4 Mn deficiency increases intestinal permeability and oxidative stress in the mouse model of DSS-induced colitis. A, Colitis was induced in Mn-adequate (Mn-A) or Mn-deficient (Mn-D) mice by the administration of DSS for 6 days and then FITC-dextran was administered by oral gavage $4 \mathrm{~h}$ before sacrifice. Fluorescence was then measured in collected serum samples ( $\mathrm{n}=5$ per group). B and C, RT-PCR quantification of (B) tight junction and (C) adherens junction components in colon mucosal cells of Mn-D or Mn-A mice on Day 6 after DSS treatment ( $\mathrm{n}=4-6$ per group). D, Representative immunoblots of tight junction protein expression in purified colon epithelial cells from Mn-A or Mn-D mice at 6 days after the DSS treatment. Immunoblots of actin were used as the loading control. E, Quantification of relative protein expression after normalization with actin ( $n=3$ per group). $\mathrm{F}$, MnSOD activity was determined in mitochondria isolated from colon mucosal cells of mice after DSS injury. $\mathrm{G}, \mathrm{H}_{2} \mathrm{O}_{2}$ levels in the colon mucosa, as well as oxidative damage determined by levels of $(\mathrm{H})$ 8-isoprostane in colon mucosa or (I) 8-OHdG in serum, were measured in Mn-adequate (Mn-A) or Mn-deficient (Mn-D) mice on 6 days after the DSS treatment ( $\mathrm{n}=3-5 / \mathrm{group})$. Data represent means \pm SEM. $* P<.05 ; * * P<.01 ; * * * P<.001$

(Figure 4I), indicating that Mn protects against oxidative stress-associated DNA damage in this mouse colitis model. Mn deficiency, therefore, reduced cellular MnSOD activity and increased oxidative stress in the mouse model of DSSinduced colitis.

\section{5 | Dietary Mn alterations did not affect the microbiome composition in the colon}

Diet is an important mediator of the microbial community structure. $^{22}$ IBD is associated with a perturbation of gut microbiota (dysbiosis) characterized by reduced bacterial diversity. These changes include a reduced abundance of bacteria of the phyla Bacteroidetes and Firmicutes and enrichment of bacteria of the family Enterobacteriaceae. ${ }^{23}$ Dysbiosis is a key factor driving inflammation in IBD. ${ }^{24}$ However, the effect of dietary Mn on gut microbiota and the contribution of dietary Mn on the development of dysbiosis have yet to be explored. Here, we determined whether dietary $\mathrm{Mn}$ alterations can alter the microbiome composition in the colon. To ensure a uniform and normalized microbiota composition, we first co-housed 4-week-old mice and fed them a Mn-adequate diet (35 mg/kg Mn) for 28 days. 
We then divided the mice into groups and fed them either a Mn-deficient ( $<0.01 \mathrm{mg} / \mathrm{kg} \mathrm{Mn})$, Mn-adequate (35 mg/ $\mathrm{kg} \mathrm{Mn}$ ), or Mn-supplemented (300 mg/kg Mn) diet for a further 14 days (Figure 5A). No signs of toxicity were observed during this period.

We examined the effect of dietary Mn on the gut microbiota by collecting feces at the baseline (Day 0) and the end of the Mn manipulation (Day 14) and then determining the microbiota composition by Illumina $16 \mathrm{~S}$ rRNA gene sequencing. The $\alpha$ diversity, as an indicator of species diversity in each sample, was determined based on Chao, Shannon diversity, and the evenness indexes, as well as the OTU richness, were similar among the three groups at Day 0 and Day 14 (Figure 5B,C, see Table S3). The $\beta$-diversity,

(A)

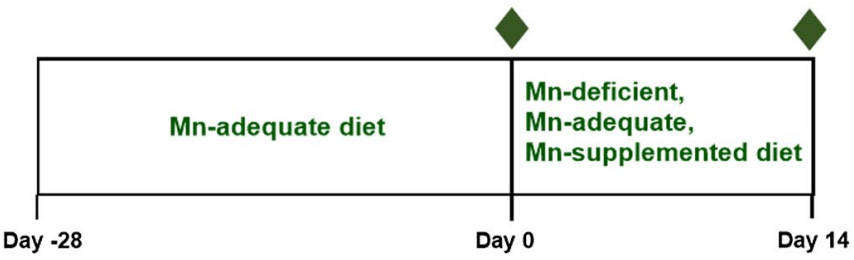

(B)

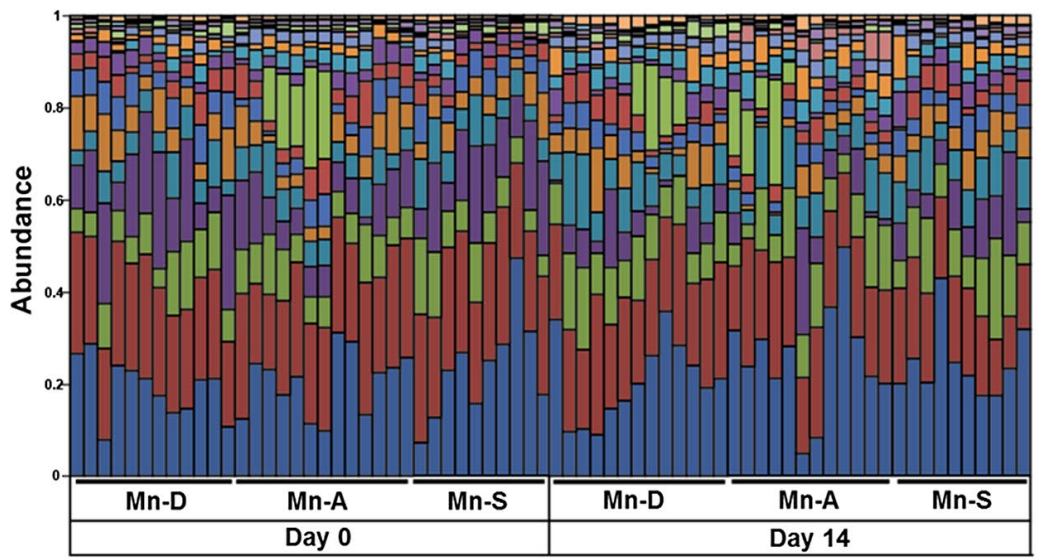

$\square$ other

$\square$ unclassified

$\square$ unclassified

Lactobacillales

Lactobaciliales

Bacteroidales

Lactobacillales

Bifidobacteriales

Bacteroidales

Bacteroidales

Clostridiales

$\square$ Bacteroidales

Bacteroidales

$\checkmark$ Clostridiales

$\checkmark$ Verrucomicrobiales

Clostridiales

Bacteroidales

$\square$ Erysipelotrichales

(C)
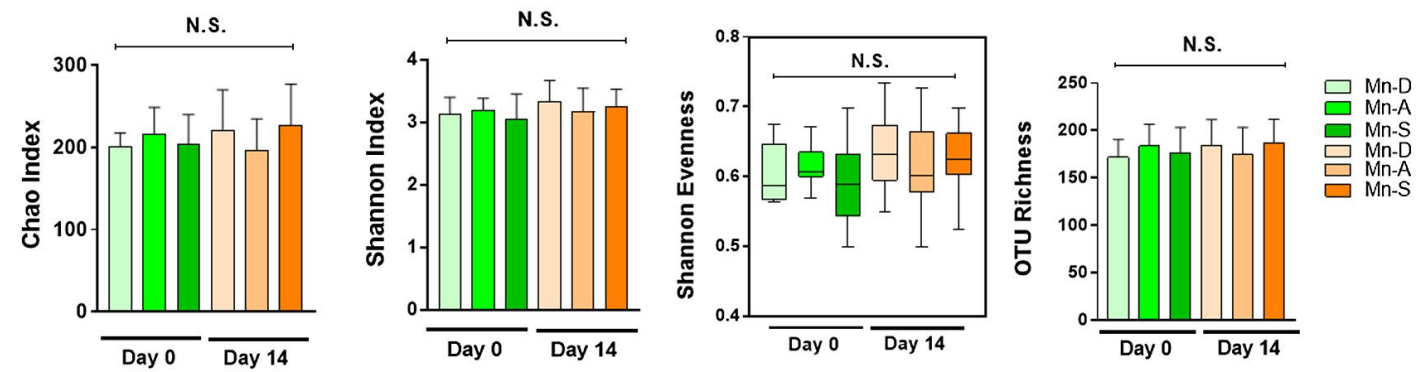

(D)

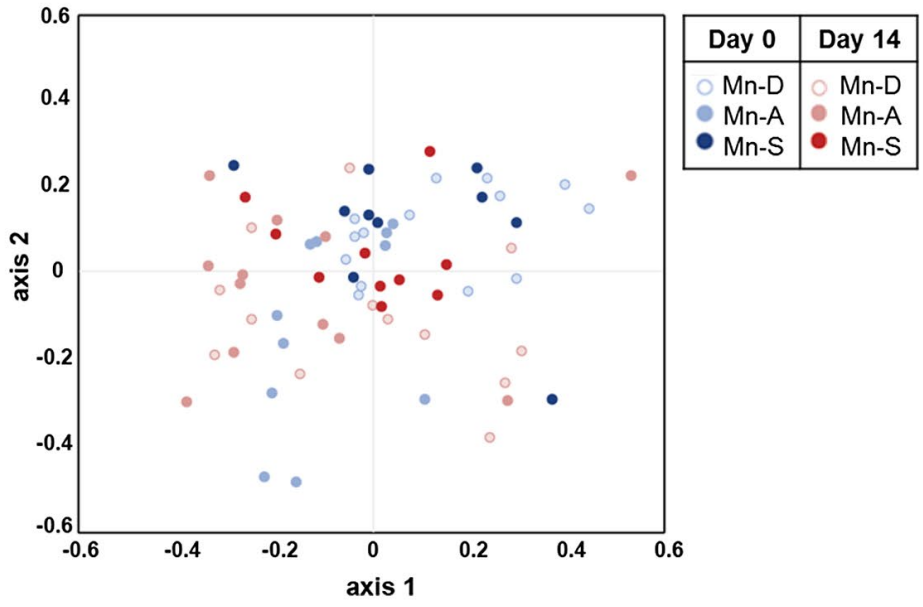


F I G U RE 5 Effect of dietary Mn alterations on the gut microbiota. A, Schematic of dietary Mn alterations. Four-week-old mice were co-housed and fed a Mn-adequate diet (35 mg/kg Mn) for 28 days and then fed with either Mn-deficient, Mn-adequate, or Mn-supplemented diets $(0,35$, or $300 \mathrm{mg} / \mathrm{kg} \mathrm{Mn}$, respectively) for 14 days. The composition of the fecal microbiota was analyzed on Day 0 and Day 14 (indicated by green diamonds) ( $\mathrm{n}=10-12$ per group). $\mathrm{B}$, Relative abundance of dominant bacterial families in feces collected from mice fed different diets on Day 0 and Day 14. C, The $\alpha$ diversity, including Chao, Shannon diversity, Shannon evenness, and OTU richness indexes, of fecal microbiota composition from mice fed different diets on Day 0 and Day 14. Data represents means \pm SEM for Chao, Shannon diversity, and OTU richness indexes. Boxes represent the interquartile range between the first and third quartiles (25th and 75th percentiles, respectively), the horizontal line inside the box defines the median, and whiskers represent the lowest and highest values for Shannon evenness. NS = not significant; the Shapiro-Wilk normality test, followed by the Kolmogorov-Smirnov test, normality test. D, NMDS plot of $\beta$-diversity values $\left(\theta_{\mathrm{YC}}\right.$ indexes) of microbiota in fecal pellets from mice fed different diets on Day 0 and Day 14 is shown with the minimal stress (stress $=0.144335$ ) after 1,000 iterations ( $\mathrm{n}=10$ per group). Overall, no significant difference was noted in $\beta$-diversity between microbiota between different groups on Day 0 and Day 14 with $P>.05$ (permutational multivariate analysis of variance [PERMANOVA]). See Tables S4-S6

determined by the theta $\mathrm{YC}\left(\theta_{\mathrm{YC}}\right)$ and Bray-Curtis dissimilarity index $(\mathrm{BC})$, which measure the diversity in the microbial community, showed no significantly distinct fecal microbiota differences in the three mouse groups at Day 0 and Day 14 (Figure 5D, see Table S4). The abundance of individual OTUs in the fecal microbiota did not significantly differ between the three groups, as determined by linear discriminant analysis effect size (LEfSe) analysis, followed by false discovery rate and family-wise error rate analyses $(q>0.05)$ (see Tables S5 and S6). These results indicate that dietary $\mathrm{Mn}$ alterations did not significantly change the gut microbiome composition in mice fed diets differing in their concentrations $(0,35$, and $300 \mathrm{mg} / \mathrm{kg})$ of $\mathrm{Mn}$.

\section{6 | Dietary Mn deficiency predisposes the colon to impairment of epithelial tight junction functions and oxidative damage}

In our experimental paradigm, mice were fed a $\mathrm{Mn}$ deficient diet two weeks prior to the induction of DSSmediated colitis (Figure 1A). Mn deficiency increased the appearance of key pathological hallmarks of IBD (ie, Mn deficiency increased inflammation, oxidative stress, and epithelial permeability); however, the possibility remained that dietary Mn deficiency itself could influence these parameters, thereby predisposing animals to a more severe form of colitis. We, therefore, examined the effects of $\mathrm{Mn}$ deficiency alone, without DSS treatment, on epithelial tight junction functions.

Mn-deficient mice showed significantly increased expression of the chemokines $C c l 2$ and $C x c l l$, but showed no significant change in cytokines $T n f \alpha, I l 6, I l 1 \beta$, and $I l 10$ (Figure 6A). The FITC-dextran experiments confirmed that a Mn-deficient diet alone could increase colon tissue permeability (Figure $6 \mathrm{~B})$. We also found substantial reductions in the gene expression of tight junction components, such as Zo1, Zo2, Cldn2, and $C l d n 3$, in the colon mucosa of Mn-deficient mice, even in the absence of DSS treatment (Figure 6C). Unlike the effects observed in DSS-treated mice (Figure 4B), occludin expression was not altered by Mn deficiency. No changes were detected in the gene expression of the adherens junction components Cdhl and Nectinl (Figure 6D). Immunoblot analysis confirmed the diminished levels of the tight junction proteins ZO1, ZO2, CLDN2, but not of OCLN, in Mn-deficient mice (Figure 6E,F).

Measurements of key parameters of oxidative damage in vivo revealed a significantly lower MnSOD activity in the colons of Mn-deficient mice than in Mn-adequate mice (Figure 6G). Similarly, $\mathrm{H}_{2} \mathrm{O}_{2}$ production (Figure $6 \mathrm{H}$ ) and the levels of the oxidative stress marker 8-isoprostane were significantly higher in the colons of Mn-deficient mice (Figure 6I). Comparison of these measurements with the DSS-induced IBD model (Figure 4) indicated that DSS treatment exacerbated the oxidative stress caused by $\mathrm{Mn}$ deficiency. Taken together, these findings indicate that dietary Mn deficiency itself impairs the intestinal epithelial tight junctions and increases oxidative damage, thereby predisposing the colon to intestinal injury and inflammation.

\section{7 | Mn supplementation increased tolerance in response to DSS treatment}

We examined the role of $\mathrm{Mn}$ in colitis in greater detail by investigating whether dietary Mn supplementation alters the susceptibility to DSS-induced injury. Mice were fed either a Mn-adequate (35 mg/kg Mn) or Mn-supplemented (300 mg/ $\mathrm{kg} \mathrm{Mn}$ ) diet for 14 days, followed by treatment with 3\% DSS in the drinking water to induce colitis (inflammatory phase) (Figure 7A). The mice were then placed on regular drinking water for 7 days (recovery phase) (Figure 7A). During the monitoring phases, the mice continued to receive the same Mn diet. Over the period of DSS treatment, the body weights of the two groups did not differ significantly 


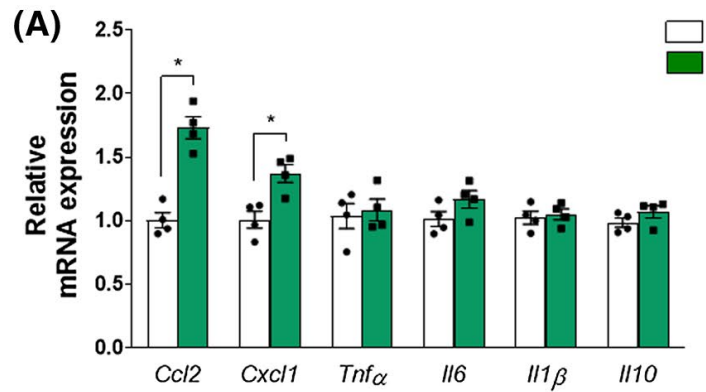

(B)
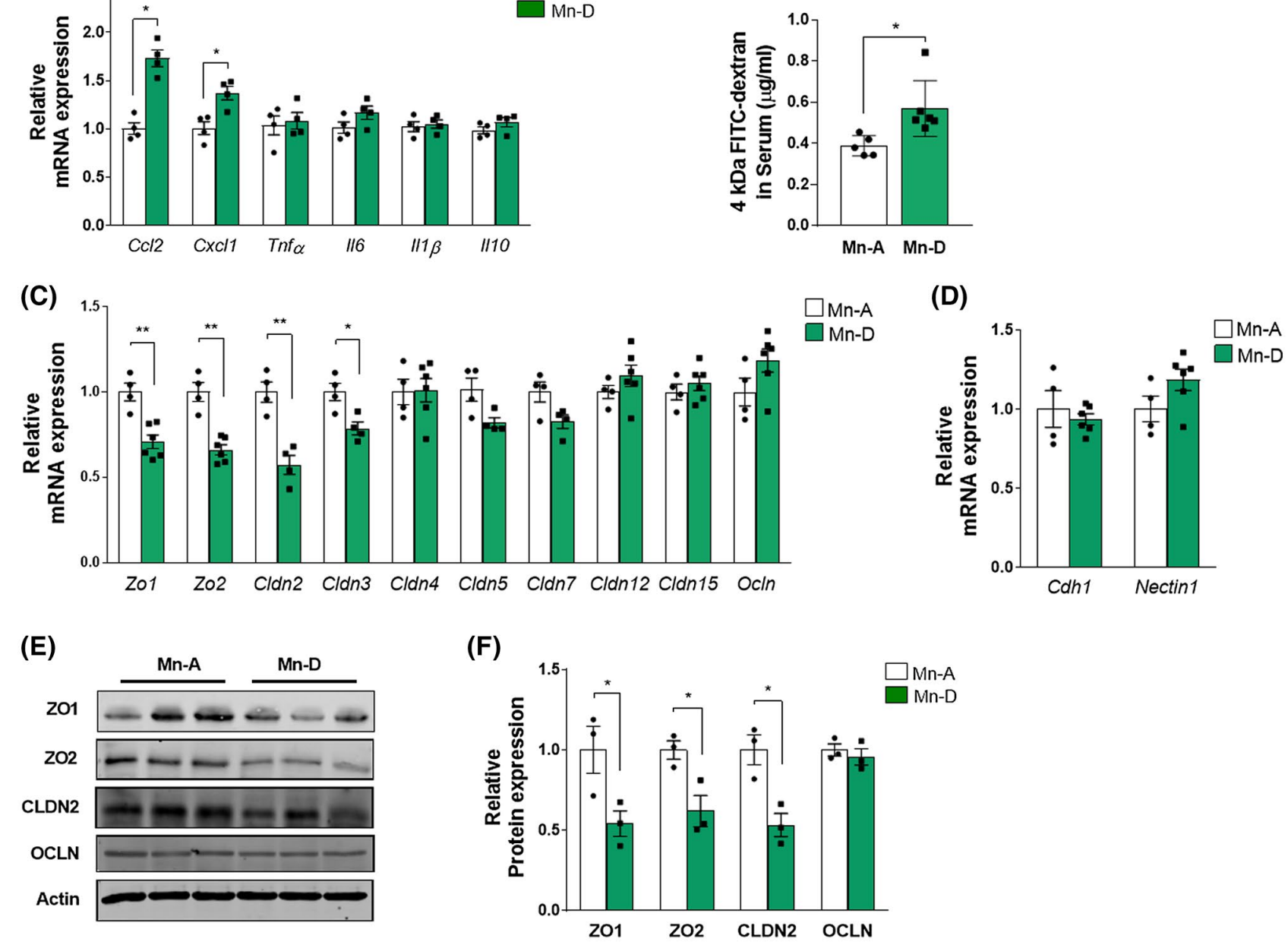

(G)

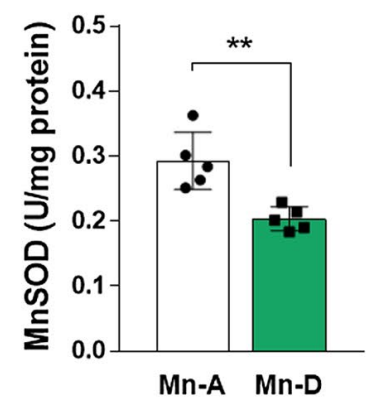

(H)

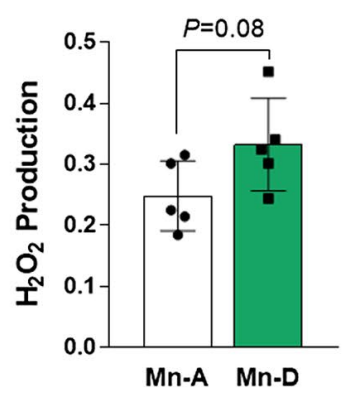

(I)

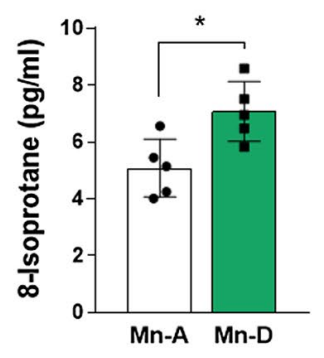

FI G U RE 6 Intestinal permeability and oxidative stress increase with dietary Mn deficiency. A, RT-PCR quantification of proinflammatory cytokines and chemokines in colon mucosa from Mn-adequate (Mn-A) or Mn-deficient (Mn-D) mice on Day 14 ( $n=4$ per group). B, Mice fed either Mn-A or Mn-D diets for 2 weeks were administered FITC-dextran by oral gavage $4 \mathrm{~h}$ before euthanasia. Serum was prepared and fluorescence was measured ( $\mathrm{n}=5-6$ mice per group). C and D, RT-PCR quantification of (C) tight junction and (D) adherens junction components in colon mucosa ( $n=4-6$ mice per group). E, Representative immunoblots of tight junction protein expression in purified colon epithelial cells. Actin was used as a loading control. F, Quantification of relative protein expression after normalization with actin ( $\mathrm{n}=3$ per group). G, MnSOD activity was determined in mitochondria isolated from colon mucosal cells in Mn-adequate (Mn-A) or Mn-deficient (Mn-D) mice. $\mathrm{H}, \mathrm{H}_{2} \mathrm{O}_{2}$ levels, as well as (I) oxidative damage determined by 8-isoprostane levels in the colon mucosa were measured from in Mn-A or Mn-D mice ( $\mathrm{n}=5$-6 per group). Data represent means \pm SEM. $* P<.05 ; * * P<.01$

$(P=.22$, Figure 7B). However, significantly longer colon lengths were observed in the Mn-supplemented mice than in the Mn-adequate mice (Figure 7C), suggesting an alleviation of DSS-induced colitis. These data indicate that dietary Mn supplementation may be slightly protective against DSSinduced experimental colitis. 


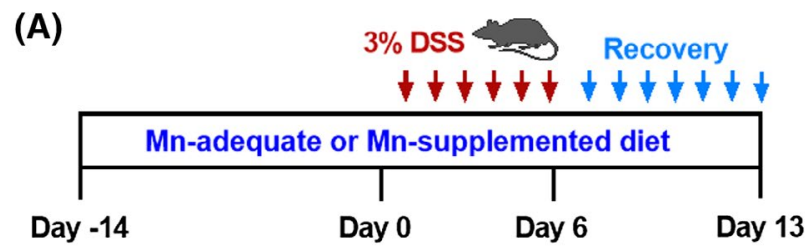

(B)

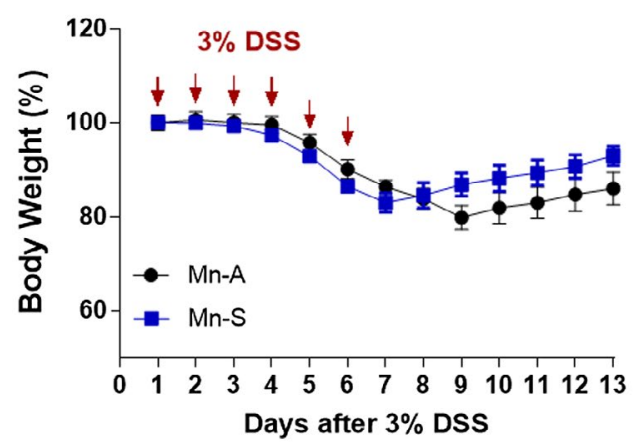

(C)

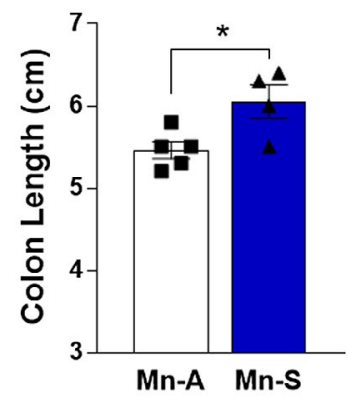

F I G U RE 7 Dietary Mn supplementation increases tolerance in response to DSS treatment. A, Schematic of the DSS-induced colitis model. Mice were fed with either Mn-adequate (Mn-A) or Mn-supplemented (Mn-S) diets (35 and $300 \mathrm{mg} / \mathrm{kg} \mathrm{Mn}$, respectively). For colitis induction, mice were provided with drinking water containing 3\% (w/v) DSS for 6 days (inflammatory phase). The mice were then placed on regular drinking water for 7 days (recovery phase). B, Body weight changes over time. Mice were treated with 3\% DSS in drinking water for 6 days, followed by 7 days of regular drinking water. Body weight was measured over the entire 13 day period ( $\mathrm{n}=15$ per group). C, Colon length from mice fed Mn-adequate (Mn-A) or Mn-supplemented (Mn-S) diets on Day 6 after DSS treatment $(\mathrm{n}=4-5$ per group). Data represent means \pm SEM. $* P<.05$

\section{8 | Discussion}

This study provides the first evidence that dietary Mn deficiency exacerbates intestinal injury and inflammation in an experimental colitis model. Our results provide some mechanistic insights into IBD, as they revealed how dietary $\mathrm{Mn}$ can maintain the intestinal barrier integrity and limit the development of colitis during colon injury. Micronutrient deficiencies have been, for decades, a major concern in the management of IBD as numerous studies have documented associations between IBD and deficiencies in trace minerals, such as $\mathrm{Fe}, \mathrm{Zn}$, and $\mathrm{Se}^{25} \mathrm{Fe}$ deficiency is associated with the bleeding, malabsorption, or anemia that occurs in one-third of IBD patients, ${ }^{26}$ while zinc deficiency is also common in patients with IBD (15 to $40 \%$ of the patient population). ${ }^{27}$ Similarly, an inverse association has been reported for nutritional Se status and the occurrence of IBD. ${ }^{28}$ These previous studies are reminiscent of the findings described here for $\mathrm{Mn}$, as deficiencies in these other trace minerals also show effects on the intestinal barrier and immune functions. ${ }^{25}$

One striking feature of Mn deficiency was its impact on the severity of DSS-induced colitis. Previous studies using Se-deficient diets $(<0.01 \mathrm{mg} / \mathrm{kg})$ showed that a time period of 12 weeks was required before Se deficiency resulted in any worsening in the colons of DSS-induced colitis in mice. ${ }^{29}$ In contrast, rats fed a zinc-deficient diet $(5 \mathrm{mg} / \mathrm{kg})$ for 3 weeks showed exacerbation of DSS-induced colitis. ${ }^{30}$ In the present study, dietary Mn deficiency worsened the severity of experimental colitis and promoted IBD development in mice after feeding a low Mn diet for only 2 weeks, and the DSS treatment often led to death of the Mn-deficient animals (Figure 2). Our data underscore the crucial role of dietary $\mathrm{Mn}$ in maintaining intestinal mucosal homeostasis and suppressing IBD.

DSS-induced colitis resembles human ulcerative colitis with respect to the impaired barrier function. ${ }^{31}$ DSS treatment of mice causes epithelial cell injury and a resulting immune response that can alter the mucosal barrier function throughout the colon epithelium ${ }^{32}$ and increase mucosal permeability. ${ }^{33}$ The DSS model is, therefore, an excellent model for investigating the effects of Mn on mucosal barrier function. Our use of this model confirmed that Mn-deficient mice displayed an increased intestinal permeability and impaired expression of tight junction proteins when compared to $\mathrm{Mn}$-adequate mice. Strikingly, even in the absence of DSS treatment, mice fed with a Mn-deficient diet displayed a 1.5 -fold greater permeability and impaired expression of tight junction proteins when compared with mice fed a Mnadequate diet (Figure 6). These data suggest that impairment of the intestinal barrier in response to a Mn-deficient diet makes the mice more susceptible to severe intestinal injury 
and inflammation following DSS administration. Studies are currently underway using acute and chronic colitis models to define the crucial role of Mn in IBD and to identify the mechanism by which Mn drives inflammation and compromises the intestinal barrier function.

Diet affects the composition of the human gut microbiome and its microbial metabolites. ${ }^{34}$ The gut microbiome plays a crucial role in the pathogenesis of IBD; for example, infusion of intestinal luminal contents into an excluded ileum triggers postoperative recurrence in Crohn's disease patients. ${ }^{35}$ Microbial colonization is required for the development of active inflammation in the mouse model of DSSinduce colitis and in IL-10 deficient mice. ${ }^{36,37}$ Increased dietary heme $\mathrm{Fe}$ intake by mice has also been associated with marked changes in the composition of the gut microbiota. ${ }^{38}$ In contrast, depletion of luminal Fe can alter the gut microbial composition and trigger inflammation in a mouse model of Crohn's disease. ${ }^{39}$ Our study is the first to assess whether alterations in dietary $\mathrm{Mn}$ alone are associated with changes in the composition of gut microbiota and whether these changes affect intestinal permeability and the development of intestinal inflammation. We found that feeding diets containing different concentrations of $\mathrm{Mn}(0,35$, or $300 \mathrm{mg} / \mathrm{kg} \mathrm{Mn})$ for 2 weeks did not change the composition of the mouse gut microbiome. One possibility is that a 2 -week period of dietary Mn alteration may be insufficient to induce changes in the composition of the gut microbiota. Future studies should address whether a longer duration of dietary Mn alteration can change the microbiome composition.

Interestingly, $\mathrm{Fe}, \mathrm{Zn}$, and Se are abundant in animal foods, such as red meats, seafood, and poultry, whereas $\mathrm{Mn}$ is essentially absent from these foods and must be obtained from plant sources. As society has become industrialized, foods associated with the typical Western diet, characterized by high intakes of red meat, sugary desserts, high-fat foods, and refined grains, have replaced traditional plant-based, and therefore, Mn-adequate diets, leading to consumption of foods low in $\mathrm{Mn} .{ }^{7}$ Epidemiological studies have revealed a $>40 \%$ reduction in a dietary $\mathrm{Mn}$ consumption in the past 15 years in the United States ${ }^{7,40,41}$ and a similar substantial decline in Mn consumption has been reported in China within the last 14 years ${ }^{42,43}$ and in South Korea within the last 6 years. ${ }^{44,45}$ These studies are congruent with an increasing incidence of IBD in developed countries ${ }^{46}$ and they indicate that, despite the rarity of dietary Mn deficiency (ie, the lack of $\mathrm{Mn}$ ) regardless of dietary lifestyle, a Mn insufficiency is prevalent. Our data clearly demonstrated that tissue Mn deficiency occurs in a rapid and robust manner. Therefore, we postulate that reductions in dietary Mn intake might be one of the contributing factors that would explain the observed worldwide increases in IBD incidence. Future study should address whether dietary Mn might provide dose-dependent protection against IBD.
Epidemiological data have shown lower Mn levels in pediatric patients newly diagnosed with Crohn's disease or ulcerative colitis, ${ }^{8}$ which suggests that Mn deficiency may be one of the potential risk factors for pediatric IBD. Nutrition is a critical factor in the onset and treatment of pediatric IBD. ${ }^{47}$ There is increasing evidence to suggest that dietary habits such as high consumption of animal proteins, fatty foods, and sugary desserts may predispose patients to the onset of pediatric IBD. ${ }^{47}$ Our study suggests that a Mn-rich, plant-based diet might protect the colonic mucosa against inflammation in pediatric patients with IBD. Thus, an assessment of patients' nutritional Mn status may be beneficial for making recommendations regarding dietary habits to those at a high risk of IBD, especially among the pediatric population. No formal recommended dietary allowance for Mn exists. The Institute of Medicine's Dietary Reference Intake for Mn cites $\sim 2 \mathrm{mg}$ /day as adequate for adults and $1.2-1.5 \mathrm{mg}$ /day as adequate for children. The Dietary Reference Intake also lists 9-11 mg/day of Mn for adults and 2-6 mg/day for children as the upper tolerable limit likely to pose no risk of adverse effects. ${ }^{48}$ However, the guidelines were set for healthy individuals and it remains unclear whether these numbers apply to the restoration of a severely injured gastrointestinal tract associated with low Mn in IBD patients.

Our work suggests that the restoration of nutritional $\mathrm{Mn}$ status to the normal range could be useful in the prevention and/or abrogation of IBD. One might argue that Mn toxicity can be a concern for this type of strategy; however, most Mn toxicity is triggered by the inhalation of Mn dust in occupational settings, such as is reported in workers employed in Mn dioxide mines, smelters, steel manufacturing plants, and dry-cell battery factories. ${ }^{9}$ Thus, no evidence of dietary Mn toxicity has been found in humans. ${ }^{49}$ This is because the homeostatic regulation of dietary Mn levels is tightly controlled by intestinal absorption and hepatobiliary excretion of the nutrient. ${ }^{50}$ Unlike dietary sources of $\mathrm{Mn}$, airborne $\mathrm{Mn}$ circumvents the first-pass clearance mechanisms. Therefore, inhaled Mn is effectively transported across the air-brain barrier, thereby potentially leading to brain Mn accumulation. In our Mn supplementation study, we fed mice with 300 ppm $\mathrm{Mn}$ and found a slight protective effect against DSS-induced colitis. Previous mouse studies have shown that oral supplementation with Mn even at levels of $\sim 2400 \mathrm{ppm}$ does not cause toxicity. ${ }^{51,52}$ This finding suggests that Mn toxicity would be less of a concern in the design of dietary Mn supplementation for this IBD mouse model. Future studies will be needed to determine the dietary Mn levels that would be most beneficial for human patient populations.

\section{ACKNOWLEDGEMENTS}

This work was supported by the National Institutes of Health grant nos. R00 ES024340 (Young Ah Seo), R01 DK095201 (Yatrik M. Shah), R01 DK118023 (Linda C. Samuelson), 
and R01 NS089896 (Shigeki Iwase); supported by a senior research award from the Crohn's and Colitis Foundation of America (Naohiro Inohara); and supported by University of Michigan Center for Gastrointestinal Research (UMCGR) Pilot Feasibility Program no. NIDDK 5P30DK034933 (Young Ah Seo). We thank Bernard Lo for technical assistance. Raw sequences are available via NCBI SRA with BioProject number PRJNA561381.

\section{CONFLICT OF INTEREST}

The authors declare no conflict of interest.

\section{AUTHOR CONTRIBUTIONS}

Y.A. Seo conceived and designed research; E.-K. Choi, L. Aring, N.K. Das, S. Solanki, and Y.A. Seo performed experiments; E.-K. Choi, N. Inohara, and Y.A. Seo analyzed the data; Y.A. Seo wrote the manuscript; N. Inohara, S. Iwase, L.C. Samuelson, Y.M. Shah, and Y.A. Seo contributed reagents, materials, and analysis tools.

\section{REFERENCES}

1. Colombel JF, Mahadevan U. Inflammatory bowel disease 2017: innovations and changing paradigms. Gastroenterology. 2017;152:309-312.

2. Konig J, Wells J, Cani PD, et al. Human intestinal barrier function in health and disease. Clin Transl Gastroenterol. 2016;7:e196.

3. Zanello G, Kevans D, Goethel A, Silverberg M, Tyler A, Croitoru K. Genetics and innate and adaptive immunity in IBD. Nestle Nutr Inst Workshop Ser. 2014;79:41-55.

4. Kenny EE, Pe'er I, Karban A, et al. A genome-wide scan of Ashkenazi Jewish Crohn's disease suggests novel susceptibility loci. PLoS Genet. 2012;8:e1002559.

5. Khalili H, Chan SSM, Lochhead P, Ananthakrishnan AN, Hart AR, Chan AT. The role of diet in the aetiopathogenesis of inflammatory bowel disease. Nat Rev Gastroenterol Hepatol. 2018;15:525-535.

6. Reddavide R, Rotolo O, Caruso MG, et al. The role of diet in the prevention and treatment of inflammatory bowel diseases. Acta Biomed. 2018;89:60-75.

7. Freeland-Graves JH, Mousa TY, Kim S. International variability in diet and requirements of manganese: causes and consequences. $J$ Trace Elem Med Biol. 2016;38:24-32.

8. Cho JM, Yang HR. Hair mineral and trace element contents as reliable markers of nutritional status compared to serum levels of these elements in children newly diagnosed with inflammatory bowel disease. Biol Trace Elem Res. 2018;185:20-29.

9. Horning KJ, Caito SW, Tipps KG, Bowman AB, Aschner M. Manganese is essential for neuronal health. Annu Rev Nutr. 2015;35:71-108.

10. Seo YA, Elkhader JA, Wessling-Resnick M. Distribution of manganese and other biometals in flatiron mice. Biometals. 2016;29:147-155.

11. Seo YA, Wessling-Resnick M. Ferroportin deficiency impairs manganese metabolism in flatiron mice. FASEB J. 2015;29:2726-2733.

12. Reeves PG, Nielsen FH, Fahey GC Jr. AIN-93 purified diets for laboratory rodents: final report of the American Institute of Nutrition ad hoc writing committee on the reformulation of the AIN-76A rodent diet. J Nutri. 1993;123:1939-1951.
13. Choi EK, Nguyen TT, Gupta N, Iwase S, Seo YA. Functional analysis of SLC39A8 mutations and their implications for manganese deficiency and mitochondrial disorders. Sci Rep. 2018;8:3163.

14. Choi EK, Nguyen TT, Iwase S, Seo YA. Ferroportin disease mutations influence manganese accumulation and cytotoxicity. FASEB J. 2019;33:2228-2240.

15. Caruso R, Ono M, Bunker ME, Nunez G, Inohara N. Dynamic and asymmetric changes of the microbial communities after cohousing in laboratory mice. Cell Rep. 2019;27(3401-3412):e3403.

16. Eichele DD, Kharbanda KK. Dextran sodium sulfate colitis murine model: An indispensable tool for advancing our understanding of inflammatory bowel diseases pathogenesis. World J Gastroenterol. 2017;23:6016-6029.

17. Antoni L, Nuding S, Wehkamp J, Stange EF. Intestinal barrier in inflammatory bowel disease. World $J$ Gastroenterol. 2014;20:1165-1179.

18. Lechuga S, Ivanov AI. Disruption of the epithelial barrier during intestinal inflammation: quest for new molecules and mechanisms. Biochim Biophys Acta Mol Cell Res. 2017;1864:1183-1194.

19. Trivedi PP, Jena GB. Ulcerative colitis-induced hepatic damage in mice: studies on inflammation, fibrosis, oxidative DNA damage and GST-P expression. Chem Biol Interact. 2013;201:19-30.

20. Van't Erve TJ, Lih FB, Jelsema C, et al. Reinterpreting the best biomarker of oxidative stress: The 8-iso-prostaglandin F2alpha/prostaglandin F2alpha ratio shows complex origins of lipid peroxidation biomarkers in animal models. Free Radic Biol Med. 2016;95:65-73.

21. Yahia D, Haruka I, Kagashi Y, Tsuda S. 8-Hydroxy-2'deoxyguanosine as a biomarker of oxidative DNA damage induced by perfluorinated compounds in TK6 cells. Environ Toxicol. 2016;31:192-200.

22. Rapozo DC, Bernardazzi C, de Souza HS. Diet and microbiota in inflammatory bowel disease: The gut in disharmony. World $J$ Gastroenterol. 2017;23:2124-2140.

23. Matsuoka K, Kanai T. The gut microbiota and inflammatory bowel disease. Semin Immunopathol. 2015;37:47-55.

24. Zuo T, Ng SC. The gut microbiota in the pathogenesis and therapeutics of inflammatory bowel disease. Front Microbiol. 2018;9:2247.

25. Gilca-Blanariu GE, Diaconescu S, Ciocoiu M, Stefanescu G. New insights into the role of trace elements in IBD. Biomed Res Int. 2018;2018:1813047.

26. Stein J, Dignass AU. Management of iron deficiency anemia in inflammatory bowel disease-a practical approach. Ann Gastroenterol. 2013;26:104-113.

27. Lomer MCE, Cahill O, Baschali A, et al. A multicentre study of nutrition risk assessment in adult patients with inflammatory bowel disease attending outpatient clinics. Ann Nutr Metab. 2019;74:18-23.

28. Kudva AK, Shay AE, Prabhu KS. Selenium and inflammatory bowel disease. Amer J Physiol Gastrointest Liver Physiol. 2015;309:G71-G77.

29. Barrett CW, Singh K, Motley AK, et al. Dietary selenium deficiency exacerbates DSS-induced epithelial injury and AOM/DSSinduced tumorigenesis. PLoS ONE. 2013;8:e67845.

30. Suwendi E, Iwaya H, Lee JS, Hara H, Ishizuka S. Zinc deficiency induces dysregulation of cytokine productions in an experimental colitis of rats. Biomed Res. 2012;33:329-336.

31. Okayasu I, Hatakeyama S, Yamada M, Ohkusa T, Inagaki Y, Nakaya R. A novel method in the induction of reliable experimental acute and chronic ulcerative colitis in mice. Gastroenterology. 1990;98:694-702. 
32. Kiesler P, Fuss IJ, Strober W. Experimental models of inflammatory bowel diseases. Cell Mol Gastroenterol Hepatol. 2015;1:154-170.

33. Kitajima S, Takuma S, Morimoto M. Changes in colonic mucosal permeability in mouse colitis induced with dextran sulfate sodium. Exp Anim. 1999;48:137-143.

34. Kau AL, Ahern PP, Griffin NW, Goodman AL, Gordon JI. Human nutrition, the gut microbiome and the immune system. Nature. 2011;474:327-336.

35. D'Haens GR, Geboes K, Peeters M, Baert F, Penninckx F, Rutgeerts P. Early lesions of recurrent Crohn's disease caused by infusion of intestinal contents in excluded ileum. Gastroenterology. 1998;114:262-267.

36. Sellon RK, Tonkonogy S, Schultz M, et al. Resident enteric bacteria are necessary for development of spontaneous colitis and immune system activation in interleukin-10-deficient mice. Infect Immun. 1998;66:5224-5231.

37. Hudcovic T, Stepankova R, Cebra J, Tlaskalova-Hogenova H. The role of microflora in the development of intestinal inflammation: acute and chronic colitis induced by dextran sulfate in germ-free and conventionally reared immunocompetent and immunodeficient mice. Folia Microbiol (Praha). 2001;46:565-572.

38. IJssennagger N, Derrien M, van Doorn GM, et al. Dietary heme alters microbiota and mucosa of mouse colon without functional changes in host-microbe cross-talk. PLOS ONE. 2012;7:e49868.

39. Werner T, Wagner SJ, Martinez I, et al. Depletion of luminal iron alters the gut microbiota and prevents Crohn's disease-like ileitis. Gut. 2011;60:325-333.

40. Hallfrisch J, Powell A, Carafelli C, Reiser S, Prather ES. Mineral balances of men and women consuming high fiber diets with complex or simple carbohydrate. J Nutri. 1987;117:48-55.

41. Egan SK, Tao SS, Pennington JA, Bolger PM. US food and drug administration's total diet study: intake of nutritional and toxic elements, 1991-96. Food Addit Contam. 2002;19:103-125.

42. Association $\mathrm{CN}$. Chinese dietary reference intakes. Beijing: ChineseLight Industry Press; 2001.

43. Jiang J, Lu S, Zhang H, et al. Dietary intake of human essential elements from a total diet study in Shenzhen, Guangdong province, China. J Food Compos Anal. 2015;39:1-7.

44. Kim E-Y, Bae Y-J, Kim S-J, Choi M-K. Estimation of manganese daily intake among adults in Korea. Nutri Res Pract. 2008;2:22-25.
45. Kang M, Kim DW, Baek YJ, et al. Dietary supplement use and its effect on nutrient intake in Korean adult population in the Korea National Health and Nutrition Examination Survey IV (20072009) data. Eur J Clin Nutr. 2014;68:804-810.

46. Ng SC, Shi HY, Hamidi N, et al. Worldwide incidence and prevalence of inflammatory bowel disease in the 21 st century: a systematic review of population-based studies. Lancet. 2018;390:2769-2778.

47. Penagini F, Dilillo D, Borsani B, et al. Nutrition in pediatric inflammatory bowel disease: from etiology to treatment. A systematic review. Nutrients. 2016;8(6):334.

48. Trumbo P, Yates AA, Schlicker S, Poos M. Dietary reference intakes: vitamin A, vitamin $\mathrm{K}$, arsenic, boron, chromium, copper, iodine, iron, manganese, molybdenum, nickel, silicon, vanadium, and zinc. J Am Diet Assoc. 2001;101:294-301.

49. Finley JW, Penland JG, Pettit RE, Davis CD. Dietary manganese intake and type of lipid do not affect clinical or neuropsychological measures in healthy young women. $J$ Nutri. 2003;133:2849-2856.

50. Aschner JL, Aschner M. Nutritional aspects of manganese homeostasis. Mol Aspects Med. 2005;26:353-362.

51. Jenkitkasemwong S, Akinyode A, Paulus E, et al. SLC39A14 deficiency alters manganese homeostasis and excretion resulting in brain manganese accumulation and motor deficits in mice. Proc Natl Acad Sci USA. 2018;115:E1769-E1778.

52. Xin Y, Gao H, Wang J, et al. Manganese transporter Slc39a14 deficiency revealed its key role in maintaining manganese homeostasis in mice. Cell Discov. 2017;3:17025.

\section{SUPPORTING INFORMATION}

Additional supporting information may be found online in the Supporting Information section.

How to cite this article: Choi E-K, Aring L, Das NK, et al. Impact of dietary manganese on experimental colitis in mice. The FASEB Journal. 2020;34:29292943. https://doi.org/10.1096/fj.201902396R 